\title{
The characteristics and pre-hospital management of blunt trauma patients with suspected spinal column injuries: a retrospective observational study
}

\author{
J. T. Oosterwold ${ }^{1,2} \cdot$ D. C. Sagel ${ }^{2}$ P. M. van Grunsven ${ }^{3} \cdot$ M. Holla $^{4} \cdot$ \\ J. de Man-van Ginkel ${ }^{5,6} \cdot$ S. Berben ${ }^{7,8}$
}

Received: 28 July 2015 / Accepted: 28 May 2016 / Published online: 8 June 2016

(C) The Author(s) 2016. This article is published with open access at Springerlink.com

\begin{abstract}
Background Pre-hospital spinal immobilisation by emergency medical services (EMS) staff is currently the standard of care in cases of suspected spinal column injuries. There is, however, a lack of data on the characteristics of patients who received spinal immobilisation during the pre-hospital phase and on the adverse effects of immobilisation. The objectives of this study were threefold. First, we determined the pre-hospital characteristics of blunt trauma patients with suspected spinal column injuries who were immobilised by EMS staff. Second, we assessed the choices made by EMS staff regarding spinal
\end{abstract}

J. T. Oosterwold

j.oosterwold@umcg.nl

1 School of Nursing and Health, University Medical Centre Groningen, PO Box 30.001, 9700 RB Groningen, The Netherlands

2 Ambulance Department, University Medical Centre Groningen, Roden, The Netherlands

3 Ambulance Emergency Medical Service Gelderland-Zuid, Nijmegen, The Netherlands

4 Department of Orthopaedic Surgery, Radboud University Medical Centre, Nijmegen, The Netherlands

5 Department of Rehabilitation, Nursing Science and Sports, University Medical Centre Utrecht, Utrecht, The Netherlands

6 Faculty of Medicine, Clinical Health Sciences, Utrecht University, Utrecht, The Netherlands

7 Eastern Regional Emergency Healthcare Network \& IQ Scientific Centre for Quality of Healthcare, Radboud University Medical Centre, Nijmegen, The Netherlands

8 Department of Critical and Emergency Care, Knowledge Centre of Sustainable Healthcare, HAN University of Applied Sciences, Nijmegen, The Netherlands immobilisation techniques and reasons for immobilisation. Third, we researched the possible adverse effects of immobilisation.

Design A retrospective observational study in a cohort of blunt trauma patients.

Study method Data of blunt trauma patients with suspected spinal column injuries were collected from one EMS organisation between January 2008 and January 2013. Coded data and free text notes were analysed.

Results A total of 1082 patients were included in this study. Spinal immobilisation was applied in $96.3 \%$ of the patients based on valid pre-hospital criteria. In $2.1 \%$ of the patients immobilisation was not based on valid criteria. Data of $1.6 \%$ patients were missing. Main reasons for spinal immobilisation were posterior midline spinal tenderness (37.2\% of patients) and painful distracting injuries (13.5\% of patients). Spinal cord injury (SCI) was suspected in $5.7 \%$ of the patients with posterior midline spinal tenderness. A total of $15.8 \%$ patients were immobilised using non-standard methods. The reason for departure from the standard method was explained for $3 \%$ of these patients. Reported adverse effects included pain $(n=10,0.9 \%$,); shortness of breath $(n=3,0.3 \%)$; combativeness or anxiety $(n=6$, $0.6 \%)$; and worsening of pain when supine $(n=1$, $0.1 \%$ ).

Conclusion/recommendation Spinal immobilisation was applied in $96.3 \%$ of all included patients based on pre-hospital criteria. We found that consensus among EMS staff on how to interpret the criterion 'distracting injury' was lacking. Furthermore, the adverse effects of spinal immobilisation were incompletely documented in pre-hospital care reports. To provide validated information on potential symptoms of SCI, a uniform EMS scoring system for motoric assessment should be developed. 
Keywords Blunt trauma $\cdot$ Pre-hospital · Spinal column injury $\cdot$ Spinal immobilisation $\cdot$ Emergency medical services $\cdot$ Retrospective observational study

\section{Introduction}

Patients who have suffered blunt trauma resulting in spinal column injuries, such as spinal fractures or dislocations, are at risk of developing iatrogenic spinal cord injury (SCI) due to physical movement or manipulation [1-5].

SCI is defined as a traumatic injury to the spinal cord that results in loss of motor and/or sensory functions [6]. In a European cohort $(n=250,584)$ of severely injured trauma patients (excluding penetrating injuries), $13.2 \%$ of immobilised patients had vertebral column injuries and $1.8 \%$ sustained a SCI $[7,8]$. It has been postulated that spinal immobilisation by emergency medical services (EMS) is required for all patients with suspected vertebral column injuries to prevent SCI after blunt trauma [1].

According to the 8th edition of the Advanced Trauma Life Support (ATLS) guidelines, spinal immobilisation should be maintained by a rigid cervical collar in combination with head blocks strapped to a spine board [9]. In the Netherlands, the EMS spinal immobilisation guidelines have been adjusted in 2002 and 2006 in accordance with the Pre Hospital Trauma Life Support (PHTLS) guidelines $[10,11]$. According to the guidelines of 2006, full spinal immobilisation is only indicated in patients who have sustained blunt trauma and show one or more of the following symptoms: neck/back pain or tenderness, altered level of consciousness, neurological deficits and evidence of intoxication or painful distracting injuries. Departure from the guidelines is allowed, however, in case of neck muscle spasms, increased pain, increase of neurological deficits, signs of increased intracranial pressure (ICP) or combativeness/resistance of the patient [11]. In these situations, the EMS staff can opt for a rigid collar only or head blocks with spine board only.

Despite the assumed beneficial effect of the method of spinal immobilisation advocated by the ATLS guidelines, there is growing evidence that immobilisation is associated with severe adverse effects including serious respiratory problems [11-13], increased ICP [14-16], delirium [17], iatrogenic pain or discomfort $[18,19]$ and possible deterioration of SCI [2, 20-23]. Furthermore, spinal immobilisation can cause a delay in transportation time to the hospital, which can negatively influence outcomes in patients with SCI [24].

A limitation of these studies is that they were mainly hospital-based and lacked a full pre-hospital description of, for example, patient characteristics, immobilisation techniques and adverse effects of spinal immobilisation. There is a paucity of data on the characteristics of patients who received pre-hospital spinal immobilisation and on the adverse effects of immobilisation that may occur during this phase. Furthermore, it is unknown whether EMS staff follows the 2006 spinal immobilisation guidelines with regard to applied techniques when taking care of patients with spinal column injuries. Our study had three main research goals. First, we aimed to determine the pre-hospital characteristics of blunt trauma patients with suspected spinal injuries that were immobilised by EMS staff. Second, we assessed the reasons for spinal immobilisation and the choices made by EMS staff regarding spinal immobilisation techniques. Third, we researched the occurrence of possible adverse effects of immobilisation during the pre-hospital phase.

\section{Materials and methods}

\section{Study design}

A retrospective observational study was performed in a cohort of blunt trauma patients.

\section{Population and setting}

The EMS of the region Gelderland-Zuid (VRGZ), the Netherlands, provides care to approximately 545,000 inhabitants from eight ambulance stations. All the 21 ambulances of VRGZ are staffed with two EMS professionals: an ambulance nurse and an ambulance driver. They will be further referred to as EMS staff in this study. They annually respond to approximately 21,000 high priority emergency ambulance calls. After every patient transport the EMS staff fills in a datasheet, which is added to the electronic patient record (EPR) of the EMS VRGZ.

\section{Data collection}

All patients from the EPR of the EMS VRGZ who were transported between 1 January 2008 and 31 December 2012 and who met the inclusion criteria described in Table 1 were included in this study. There were no changes in the spinal immobilisation protocol during the study period. The study did not require an ethical approval because of the retrospective observational design from anonymised data. The data was provided by the medical manager of VRGZ to the authors in a Microsoft Excel spreadsheet.

\section{Study population}

Trauma patients with full or partial external immobilisation after blunt trauma who received a high priority of the 
Table 1 Inclusion criteria of patients (see "Material and methods")

$\begin{aligned} & 18 \text { years or older } \\ & \text { and }\end{aligned}$
Life-threatening response call with a lights and sirens ambulance
response
$\quad$ and
Blunt trauma
$\quad$ and
Full or partial external immobilisation
$\quad$ and
Ambulance transport from accident site to the Emergency Depart-
ment

ambulance dispatch centre were included in the study. High priority was defined as an ambulance being on site within $15 \mathrm{~min}$ after the initial contact with the emergency medical dispatcher using lights and siren.

Further inclusion criteria for the patients were that patients had to be $\geq 18$ years of age and transported to either the Radboud University Hospital Nijmegen (Level 1 trauma centre) or the Canisius Wilhelmina Hospital Nijmegen (Level 2 trauma centre). Exclusion criteria were inter-hospital transport and helicopter emergency medical service (HEMS) transport.

\section{Variables and measurements}

\section{Characteristics of the included patients and nature of the incidents}

Age, gender and alcohol use of the patients were included in the analysis. The nature of the incident was determined as traffic, home, sports, work-related and other.

\section{Characteristics of time intervals}

To determine the time patients were lying on the spine board before arrival at the Emergency Department (ED), the following time intervals were measured: on-scene time (OST), transportation time (TrT) and the total time (TT) from the initial dispatch until arrival at the hospital. Time values were written in the form hh:mm:ss.

\section{Characteristics of suspected injury}

Suspected injuries to the spinal column or spinal cord that were coded by EMS staff were determined. To define signs of high ICP, free text notes were screened for the following items according to the PHTLS literature: evidence of head trauma combined with pupillary changes (sluggish or non-reactive), hemiparesis, hemiplegia or a Glascow Coma Scale (GCS) $<14$ [10]. Evidence of possible cranial bleeding was derived from the data that was coded as 'intracranial injury' or 'subarachnoid haemorrhage'.

\section{Characteristics of consciousness and pain}

Consciousness was measured with the GCS and categorised into three groups: severe (GCS 3-8), moderate (GCS 9-12) and mild (GCS 13-14) [10]. The general level of pain experienced by the patient was measured with scores on the Numeric Rating Scale (NRS) [25]. This is an 11-point scale ranging from 0 to 10 . A score between 1 and 3 points was classified as mild pain, a score between 4 and 6 points as moderate pain and a score between 7 and 10 points as severe pain.

\section{Characteristics of administered analgesics}

The types of analgesics administered to the patients were recorded.

\section{Characteristics of reasons for spinal immobilisation}

Spinal immobilisation is indicated when patients meet criteria as mentioned in box 1 (Fig. 1, box 1) combined with a criterion from box 2 (Fig. 1, box 2) [11]. The pre-hospital distinction between a contusion, luxation and/or fracture is not always reliable and valid; therefore, we chose to combine all the injuries that may cause acute functional impairment and subdivided them into the following categories: lower extremity pain, upper extremity pain, upper chest pain, abdominal pain, hip/iliac pain and a group with combined injuries from the previous categories. Patients with abrasions only were excluded.

The techniques used for spinal immobilisation were registered. The standard method of spinal immobilisation consists of the use of a rigid cervical collar, a long backboard, head blocks and straps. The following departures from the standard method were discerned: backboard and straps only, rigid collar only, vacuum mattress and rigid collar only, manual fixation of the head and neck only and scoop stretcher and rigid collar only. The reasons given by EMS staff for departure from the standard method were also noted [10].

Spinal immobilisation outside the standard guidelines refers to full spinal immobilisation of patients only based on the mechanism of injury (Fig. 1, box 1). These patients did not meet the criteria mentioned in box 2 . 
Fig. 1 Dutch National Protocol Ambulance Care 2006 (derived from the Pre-hospital Spinal Immobilisation Guidelines 2002)

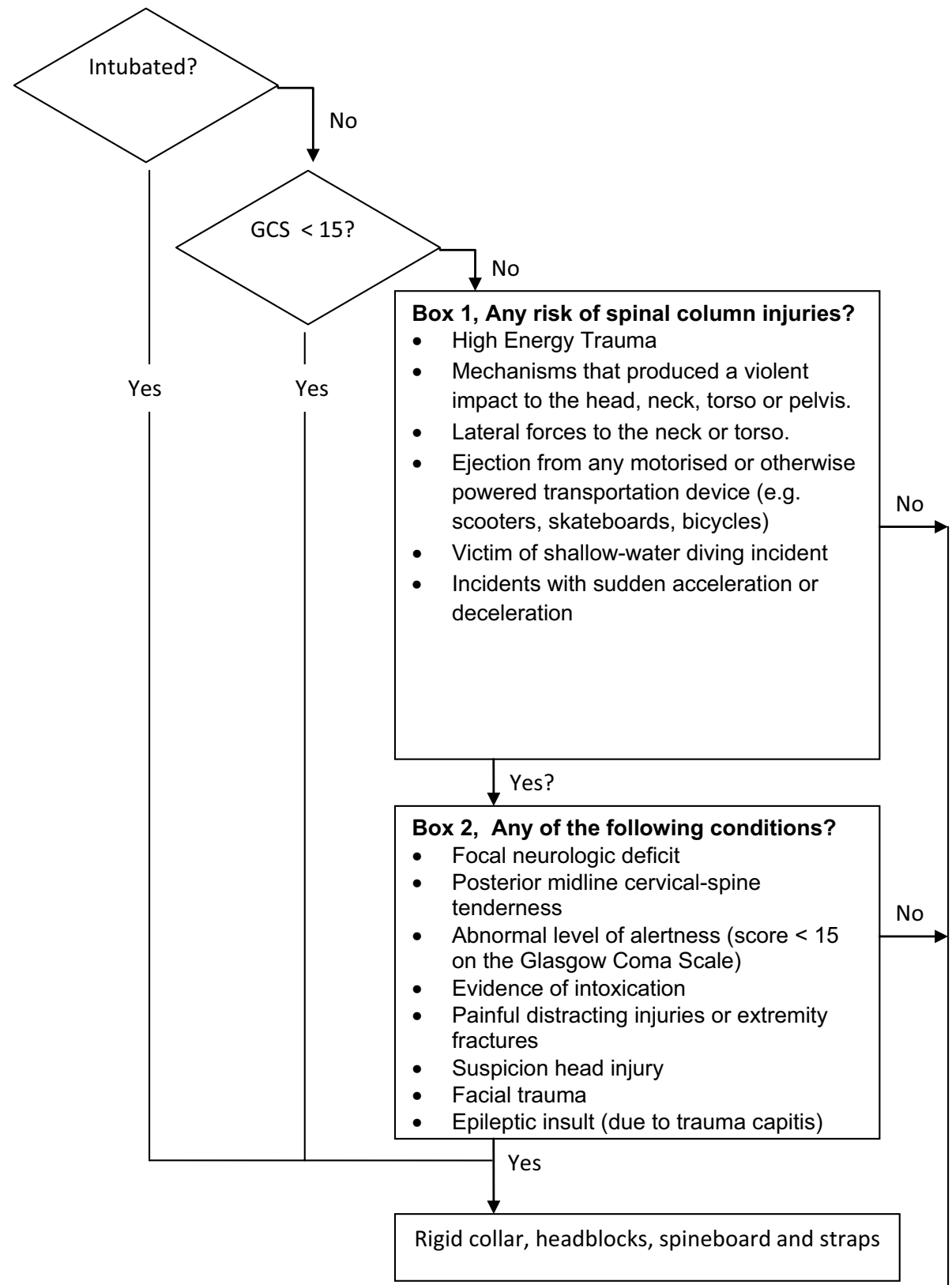

No spinal immobilisation

\section{Adverse effects}

All known adverse effects of spinal immobilisation that could be measured and detected by the EMS staff in the pre-hospital setting were registered. These effects include pain or discomfort as a result of spinal immobilisation [18], shortness of breath and a subsequent reduction in respiratory function [26] as a result of lying supine, vomiting or nausea in a moving ambulance and combativeness.

\section{Data analysis}

Coded categorical data were presented in absolute numbers and percentages. The mean and standard deviations (SD) were calculated for continuous variables. Measured time was presented in minutes and seconds. Manifest content analysis was used to analyse the free text notes [27]. This quantitative research method was used to count the frequency of the following pre-defined 
characteristics reported in the free text: use of alcohol, symptoms of high intracranial pressure, nausea or emesis, adverse effects of spinal immobilisation, methods of spinal immobilisation and criteria for spinal immobilisation.

Differences between groups were calculated with Chisquare for categorical data, and one-way ANOVA was used to test differences between several groups. $\mathrm{P}$ values of $\leq 0.05$ were considered as significant for all tests. IBM SPSS statistics version 20.0 was used to analyse the data.

\section{Results}

A total of 1082 patients received spinal immobilisation in the region Gelderland-Zuid between January 2008 and December 2012. A total of 654 (60.4\%) patients were transferred to a Level 1 trauma centre and 428 (39.6\%) patients to a Level 2 trauma centre.

\section{Characteristics of the included patients and type of incident}

The mean age of the included patients was 43 years [SD \pm 18.3 (range 18-93 years)]. Patients aged 65 years and older represented $14 \%(n=151)$ of the total study population. The male-female ratio was 643-439 (59$41 \%)$. Alcohol use was described by EMS staff in 129 (11.9\%) patients. Crash/collision incidents were coded in $209(19.3 \%)$ patients as the cause of injury. In 754 $(69.7 \%)$ patients the type incident was not coded by EMS staff.

\section{Characteristics of time interval}

The mean on scene time (OST) was 25.33 (SD 10.22) and mean transport time (TrT) was 14.24 (SD 8.14) (Table 4). The mean total time (TT) between initial contact with the emergency dispatcher and the arrival at the hospital was 49.13 (SD 16.25).

\section{Characteristics of suspected injury}

In $402(37.2 \%)$ patients the EMS staff suspected a spinal column injury, and $62(5.7 \%)$ patients demonstrated symptoms of SCI. Other suspected injuries are described in Table 2. Signs of increased ICP were documented in 75 $(6.9 \%)$ patients.

\section{Characteristics of consciousness and pain}

Severe loss of consciousness (GCS 3-8) at arrival of the ambulance was found in $61(5.6 \%)$ patients and moderate loss of consciousness (GCS 9-12) in 66 (6.1\%) patients. A total of $919(84.9 \%)$ patients were alert (GCS of 14 or 15 ). Scores of $36(3.3 \%)$ patients were missing.

In $771(71.3 \%)$ patients the EMS staff did not report the NRS. The pain intensity reported by the remaining 311 $(28.7 \%)$ patients at arrival of the ambulance varied: 200 $(18.5 \%)$ patients reported no pain, $24(2.2 \%)$ mild pain, $37(3.4 \%)$ moderate pain and $50(4.6 \%)$ severe pain. Mean pain score (NRS) in the study population was 2.09 . A second assessment of pain intensity (at arrival at ED) was missing in $1008(93.2 \%)$ patients and therefore excluded from further analysis. Results of the pain assessment at arrival of the EMS staff are presented in Table 2.

\section{Characteristics of administered analgesics}

Analgesics of one type (fentanyl, ketamine, nitrous oxide/ oxygen mixture or paracetamol) were given to 229 (21.2\%) patients; fentanyl and ketamine to $15(1.4 \%)$ patients; fentanyl and paracetamol to $19(1.8 \%)$ patients and a nitrous oxide/oxygen mixture with fentanyl to $2(0.2 \%)$ patients.

\section{Reasons for spinal immobilisation}

The reasons for spinal immobilisation are described in Table 3. Midline tenderness of the spine after blunt trauma was the main reason for immobilisation $(37.2 \%, n=402)$. Painful distracting injuries came second $(13.5 \%, n=146)$. In case of a non-tender spine, upper torso injuries were considered most frequently as distracting injuries leading to full spinal immobilisation $(5 \%, n=55)$. The mean NRS score of the group of patients that were immobilised based on the criterion 'painful distracting injuries' was $3.2(n=52)$ with a mode of 0 (Table 4$)$. An analysis of variance showed that the mean pain scores on the different categories of injuries revealed a statistically significant main effect, Welch's $F(5,6.731)=7.5, P=0.0011$, indicating that not all of the injuries had the same average pain score. Post hoc comparisons, using Games-Howell post hoc procedure, showed that patients with abdominal injuries $(M=8.00, \mathrm{SD}=1.00)$ had a significantly higher average pain score than patients with upper torso injuries $(M=2.43, \mathrm{SD}=3.15)$ and patients with injuries to different body parts $(M=2.67, \mathrm{SD}=3.96)$.

Spinal immobilisation outside the standard guidelines was performed on $23(2.1 \%)$ patients. In these cases, the decision of the EMS staff to stabilise the spine was based only on the criteria shown in Fig. 1, box 1 (trauma mechanism). The remaining 236 (21.8\%) patients were immobilised based on one of the other criteria of the Dutch NPA: focal neurological deficits, evidence of intoxication, facial trauma, epileptic insult due to trauma capitis, or unknown. 
Table 2 Patient demographics and characteristics

\begin{tabular}{|c|c|c|c|c|c|c|}
\hline & \multirow[t]{2}{*}{$n$} & \multirow[t]{2}{*}{$\%$} & \multicolumn{4}{|c|}{$\begin{array}{l}\text { Pain assessment performed by EMS staff at } \\
\text { arrival }\end{array}$} \\
\hline & & & $n$ & $\%$ & Mean NRS ${ }^{\mathrm{a}}$ & $95 \%$ CI for mean \\
\hline All patients & 1082 & 100 & 311 & 28.7 & 2.09 & $1.73-2.45$ \\
\hline \multicolumn{7}{|l|}{ Gender } \\
\hline Male & 643 & 59.4 & 188 & 17.4 & 2.03 & $1.58-2.48$ \\
\hline Female & 439 & 40.6 & 123 & 11.4 & 2.18 & $1.6-2.76$ \\
\hline \multicolumn{7}{|l|}{ Age } \\
\hline 18-64 (mean, SD) & $931(43,18)$ & 86.0 & 279 & 25.8 & 2.11 & $1.73-2.49$ \\
\hline$\geq 65$ & $151(74,7)$ & 14.0 & 32 & 3.0 & 1.88 & $0.75-3.01$ \\
\hline \multicolumn{7}{|l|}{ GCS at arrival EMS } \\
\hline GCS 3-8 & 61 & 5.6 & - & - & - & - \\
\hline GCS 9-13 & 66 & 6.1 & 14 & 1.3 & 2.14 & $0-4.28$ \\
\hline GCS $14-15$ & 919 & 84.9 & 271 & 25.0 & 2.07 & $1.7-2.44$ \\
\hline \multicolumn{7}{|l|}{ Type of accident } \\
\hline Traffic & 209 & 19.2 & 56 & 5.2 & 2.18 & $1.25-3.11$ \\
\hline Home & 66 & 6.0 & 17 & 1.6 & 2.65 & $0.86-4.44$ \\
\hline Sports & 17 & 1.6 & 8 & 0.7 & 2.75 & $0.94-4.56$ \\
\hline Work & 28 & 2.6 & 7 & 0.6 & 0.86 & $0.31-2.03$ \\
\hline Other & 8 & 0.7 & 4 & 0.4 & 0.50 & $0.48-1.48$ \\
\hline Alcohol use & 129 & 11.9 & - & - & - & - \\
\hline 18-30 years & 37 & 3.4 & - & - & - & - \\
\hline $31-64$ years & 81 & 7.5 & - & - & - & - \\
\hline$\geq 65$ years & 11 & 1.0 & - & - & - & - \\
\hline Nausea or vomiting & 87 & 8.0 & - & - & - & - \\
\hline \multicolumn{7}{|l|}{ Injuries ${ }^{b, c}$} \\
\hline Suspected vertebral column injury & 402 & 37.2 & - & - & - & - \\
\hline Suspected spinal cord injury & 62 & 5.7 & - & - & - & - \\
\hline Head injury & 407 & 37.6 & - & - & - & - \\
\hline Signs of increased ICP & 75 & 6.9 & - & - & - & - \\
\hline No signs of increased ICP & 326 & 30.1 & - & - & - & - \\
\hline Jaw fracture & 6 & 0.6 & - & - & - & - \\
\hline Upper torso injury & 174 & 16.1 & 23 & 2.1 & 2.4 & - \\
\hline Abdominal injury & 40 & 3.7 & 3 & 0.3 & 8 & - \\
\hline Lower extremity injury & 74 & 6.8 & 11 & 1.0 & 3.36 & - \\
\hline Upper extremity injury & 31 & 2.8 & 3 & 0.3 & 6 & - \\
\hline Injury to different body parts & - & - & 12 & 1.1 & 2.7 & - \\
\hline
\end{tabular}

a Numeric Rating Scale

b There may be more than one injury in a patient

c Any other injury with the exception of abrasions
In our study population, $911(84.2 \%)$ patients received full spinal immobilisation (rigid collar, head blocks, spine board and straps). A total of $102(9.4 \%)$ patients were immobilised by spine board and straps only. Two (0.2\%) patients were immobilised by a semi-rigid brace only that secures the head, neck and torso (Kendrick Extrication Device ${ }^{\circledR}$ ). Four $(0.4 \%)$ other patients were immobilised by the semi-rigid brace in conjunction with a rigid cervical collar. The vacuum mattress in combination with the cervical rigid collar was used in $6(0.6 \%)$ patients. Transportation by means of scoop stretcher occurred once with the rigid collar $(0.1 \%)$ and once $(0.1 \%)$ without manual inline stabilisation. The remaining $55(5.1 \%)$ patients were immobilised by a rigid cervical collar only (Table 3 ).

Departure from the standard method of spinal immobilisation was explained by EMS staff in 32 cases (3\%) 
Table 3 Characteristics of prehospital emergency care

\begin{tabular}{|c|c|c|c|}
\hline & $n$ & $\%$ & Mean (SD) \\
\hline \multicolumn{4}{|l|}{ Time intervals } \\
\hline Time on scene ${ }^{\mathrm{a}}$ & 1055 & & $25^{\prime} 33^{\prime \prime}\left(10^{\prime} 22^{\prime \prime}\right)$ \\
\hline Time transport to $\mathrm{ER}^{\mathrm{b}}$ & 1034 & & $14^{\prime} 24^{\prime \prime}\left(8^{\prime} 14^{\prime \prime}\right)$ \\
\hline Time from dispatch to ER & 1080 & & $49^{\prime} 13^{\prime \prime}\left(16^{\prime} 25^{\prime \prime}\right)$ \\
\hline \multicolumn{4}{|l|}{ Administered analgesics } \\
\hline Fentanyl & 199 & 18.4 & \\
\hline Esketamine & 21 & 1.9 & \\
\hline Nitrous oxide/oxygen mixture & 0 & 0 & \\
\hline Paracetamol & 9 & 0.8 & \\
\hline Fentanyl and ketamine & 15 & 1.4 & \\
\hline Fentanyl and paracetamol & 19 & 1.8 & \\
\hline Fentanyl, paracetamol and ketamine & 2 & 0.2 & \\
\hline Fentanyl and nitrous oxide/oxygen & 2 & 0.2 & \\
\hline Spinal immobilisation according to the applicable guideline & 1059 & 97.9 & \\
\hline $\begin{array}{l}\text { Posterior midline spine tenderness, and/or abnormal level of alertness } \\
(\mathrm{GCS}<15) \text {, and/or focal neurological deficit, and/or facial trauma, and/ } \\
\text { or epileptic insult (due to trauma capitis) }\end{array}$ & 767 & 70.9 & \\
\hline Painful distracting injuries only & 146 & 13.5 & \\
\hline Evidence of intoxication & 129 & 11.9 & \\
\hline Unknown & 17 & 1.6 & \\
\hline Spinal immobilisation outside the applicable guidelines & 23 & 2.1 & \\
\hline No injury, only based on trauma mechanism & 23 & 2.1 & \\
\hline \multicolumn{4}{|l|}{ Method of spinal immobilisation } \\
\hline Full spinal immobilisation & 911 & 84.2 & \\
\hline Rigid collar only & 55 & 5.1 & \\
\hline Spine board with straps only & 102 & 9.4 & \\
\hline Semi rigid brace $\left(\mathrm{KED}^{\circledR}\right)$ only & 2 & 0.2 & \\
\hline Semi rigid brace $\left(\mathrm{KED}^{\circledR}\right)$ with rigid collar & 4 & 0.4 & \\
\hline Vacuum mattress with rigid collar & 6 & 0.6 & \\
\hline Scoop stretcher only & 1 & 0.6 & \\
\hline Scoop stretcher with rigid collar & 1 & 0.1 & \\
\hline
\end{tabular}

${ }^{\text {a }}$ Cases falling greatly outside of the range $(<5$ or $>60 \mathrm{~min})$ were analysed by free text and, 27 cases were deleted because of incorrectness

${ }^{b}$ Cases falling greatly outside of the range $(<60 \mathrm{~s}$ or $>45 \mathrm{~min})$ were analysed by free text, and 48 cases were deleted because of incorrectness
(Table 5). Pain from surrounding injuries was the main reason for omitting the rigid cervical collar $(n=10,0.9 \%)$, followed by combativeness or anxiety $(n=7,0.6 \%)$. The choice for omitting the spine board was mainly based on shortness of breath $(n=2,0.2 \%)$. Following PHTLS guidelines, the rigid collar should be removed in case of increased ICP. In this study population the percentage of patients who were immobilised by backboard only $(n=102,9.4 \%)$ did not differ in patients with or without signs of increased ICP $\left[\chi^{2}(1, N=1082)=1.141, P=0.286\right]$.

\section{Adverse effects}

Vomiting or nausea was described in $87(8.0 \%)$ patients. More than half of these patients $(50.6 \%)$ received the antiemetic metoclopramide. In 45 (4.5\%) patients antiemetic drugs were administered prophylactically. Other adverse effects of spinal immobilisation included pain $(n=10,0.9 \%$,$) , shortness of breath (n=3,0.3 \%)$ and anxiety/combativeness $(n=6,0.6 \%)$ (Table 6$)$.

There were no reports of progressive signs of SCI. 
Table 4 Categories of painful distracting injuries in cases of non-tender spine and GCS 15

\begin{tabular}{|c|c|c|c|c|c|c|}
\hline & $n(\%)$ & $\begin{array}{l}\text { Number of } \\
\text { NRS }^{\mathrm{a}} \text { score }\end{array}$ & $\begin{array}{l}\text { Mode NRS } \\
\text { score }\end{array}$ & Mean NRS score & Min-max & \\
\hline $\begin{array}{l}\text { Categories of distracting } \\
\text { injuries }\end{array}$ & $146(13.5)$ & 52 & 0 & 3.21 & $0-10$ & $P=0.01^{\mathrm{b}}$ \\
\hline Upper torso injury & $55(37.7)$ & 23 & 0 & 2.4 & $0-8$ & \\
\hline Injury to different body parts & $38(26)$ & 12 & 0 & 2.6 & $0-10$ & \\
\hline Lower extremity injury & $26(17.8)$ & 9 & 0 & 3.7 & $0-10$ & \\
\hline Hip, iliac injury & $10(6.8)$ & 2 & 0,3 & 1.5 & $0-3$ & \\
\hline Upper extremity injury & $9(6.2)$ & 3 & $0,8,10$ & 6 & $0-10$ & \\
\hline Abdominal injury & $7(4.8)$ & 3 & $7,8,9$ & 8 & $7-9$ & \\
\hline Other & $1(0.7)$ & - & - & - & - & \\
\hline
\end{tabular}

${ }^{a}$ Numeric rating scale

${ }^{b} P$ value from Welch test

\section{Discussion}

To our knowledge, this is the first study that gives a comprehensive overview of the characteristics and pre-hospital management of blunt trauma patients with suspected spinal column injuries.

Our first objective was to determine the pre-hospital characteristics of this category of patients. The most important findings were that the EMS staff suspected spinal column injuries in $402(37.2 \%)$ patients and that 62 (5.7\%) patients had symptoms of SCI. The EMS staff did not report the NRS in $771(71.3 \%)$ patients. Painful distracting injuries were found in $146(13.5 \%)$ patients. The standard method of immobilisation was not used in 171 $(15.8 \%)$ patients. Finally, in $22(2 \%)$ cases adverse effects were reported by the EMS. These included pain due to spinal immobilisation $(n=10,0.9 \%$,); shortness of breath $(n=3,0.3 \%)$; combativeness or anxiety $(n=6,0.6 \%)$; and worsening of pain when supine $(n=1,0.1 \%)$.

Our second objective was to assess the reasons for spinal immobilisation and the choices made by EMS staff regarding spinal immobilisation techniques The reason for spinal immobilisation was clear to the EMS staff in most cases $(n=1059,97.9 \%)$. There was a significant mechanism of injury (Fig. 1, box 1) combined with specific signs and symptoms (Fig. 1, box 2). We found that consensus on the implementation of the criterion 'distracting injury' was lacking among EMS staff. A total of 146 (13.5\%) of the patients who did not have spinal tenderness after blunt trauma were immobilised because of a painful injury. There is, however, a difference between a painful injury and a distracting injury. While a distracting injury is a criterion for spinal immobilisation according to Fig. 1, box 2, this criterion was interpreted differently by the various EMS staff. A distracting injury is defined by PHTLS as follows: long bone fractures, visceral injury requiring surgical consultation, large laceration, degloving or crush injury, large burns and any other injury producing acute functional impairment (Table 7) [10]. Although the most painful injuries may be considered as 'distracting', we could not demonstrate an association between high NRS scores at arrival of the EMS staff and distracting injuries as a reason for spinal immobilisation. The mean NRS score in the group of patients that were immobilised based on the criterion 'painful distracting injuries' was $3.2(n=52)$ with a mode of 0 .

Previous studies by Hefferman et al. [28] and Domeier et al. [29] researched whether the definition of a distracting injury could be narrowed. Hefferman et al. focused on the c-spine and showed that patients with an upper torso injury, in cases of a non-tender cervical spine, might have sustained a cervical spine injury. Domeier et al. redefined the term 'distracting injury' as: 'a suspected extremity fracture proximal to the wrist or ankle'.

There is increasing evidence that a distracting injury, as currently defined, does not affect the sensitivity of the physical examination. Konstantinides et al. [30] concluded that only the upper chest injuries may be significant enough to decrease the sensitivity of the physical examination of the cervical spine in alert and non-intoxicated patients blunt trauma patients. Furthermore, Dahlquist et al. [31] showed, that femur fractures should not be considered as distracting injuries for cervical spine assessment. Clinical examination is a sensitive screening method for thoracolumbar spine clearance in patients with distracting injuries [32].

Therefore, further research and clarification of the criterion 'distracting injury' or a narrowing of the definition (following Hefferman et al., Domeier et al.) based on pain scores combined with injury assessment is warranted. We believe this will ultimately lead to a reduction of adverse effects of spinal immobilisation and potentially to a decrease of the number of patients, that is unnecessarily exposed to X-rays. 
Table 5 Characteristics of pre-hospital emergency care

\begin{tabular}{lll}
\hline $\begin{array}{l}\text { Reasons for departure from the standard } \\
\text { method of spinal immobilisation }\end{array}$ & $n$ & $\%$ \\
\hline Omitting the rigid collar & 26 & 2.4 \\
Pain from surrounding injury & & \\
Sternum injury & 1 & \\
Clavicle fracture & 5 & \\
Ear injury & 1 & \\
Shoulder injury & 1 & \\
Jaw fracture & 2 & \\
Combativeness or anxiety & 7 & \\
Shortness of breath & 2 & \\
Non- fitting (anatomic or clothing) & 6 & \\
Lumbar pain/tenderness only & 1 & \\
Omitting the spine board & 6 & \\
Shortness of breath & 2 & \\
Worsening of pain when supine & 1 & \\
Combativeness or anxiety & 1 & \\
Unclear &
\end{tabular}

Table 6 Adverse effects of spinal immobilisation reported as free text by the EMS

\begin{tabular}{lr}
\hline & $n(\%)$ \\
\hline Pain & $10(0.9)$ \\
Shortness of breath & $4(0.4)$ \\
Anxiety/combativeness & $6(0.6)$ \\
Worsening of pain when supine & $1(0.1)$ \\
\hline
\end{tabular}

In $23(2.1 \%)$ patients spinal immobilisation was based on the mechanism of injury criteria (Fig. 1, box 1). Departure from the standard method of spinal immobilisation was found in $171(15.8 \%)$ cases. A high CPI was not a reason for removal of the rigid collar, despite the fact that $75(6.9 \%)$ of our cohort had signs of increased ICP. No statistically significant differences could be found for the application of a rigid collar between groups of patients with or without signs of high ICP. The recommendation to remove the rigid collar in case of increased ICP was made by the PHTLS in 2002 and accepted the NPA in 2006. The reason that EMS staff did not depart from the standard method of spinal immobilisation in cases of high ICP may be explained by the non-explicit naming of the removal of the rigid collar in the NPA. A lack of awareness of this guideline can cause further increase in ICP [14]. The arguments used by EMS staff to depart from the standard method of spinal immobilisation were in accordance with the Dutch NPA protocol. One exception concerned a patient who was immobilised by spine board only because of complaints of lower back pain. The patient did not complain of pain in the cervical region. We found that the documentation of reasons for departure from the standard method was inadequate. It remains unclear, for instance, why $139(12.8 \%)$ patients were not immobilised based on the standard method. Departure from the standard method is permissible if the EMS staff can substantiate this departure. Patients and other healthcare providers have reason to expect that pre-hospital treatment follows current protocols or guidelines. It is therefore vital that the reasons for departure from the standard method are adequately documented by EMS staff. Adequate documentation leads to improved transparency of pre-hospital care and is necessary when the EMS staff or organisation has to account for its decisions [33].

The third objective of our study was to research the occurrence of possible adverse effects during the pre-hospital phase. Previous research has found adverse effects of spinal immobilisation during this phase. In their study on the effects of spinal immobilisation on healthy volunteers, Kwan et al. [18] found that $55 \%$ of healthy volunteers complained of moderate to severe pain within $30 \mathrm{~min}$ after spinal immobilisation. In addition, Bauer and Kowalski [26] found that certain devices used for spinal immobilisation had restrictive effects on the pulmonary function in the healthy, non-smoking man. Since the average on-scene and transportation times exceeded $30 \mathrm{~min}$, we expected to find adverse effects of spinal immobilisation. Our findings did not correspond with previous research, however, adverse effects were found in only $22(2 \%)$ cases (pain due to spinal immobilisation ( $n=10,0.9 \%$,); shortness of breath $(n=3,0.3 \%)$; combativeness or anxiety $(n=6$, $0.6 \%)$; and worsening of pain when supine ( $n=1,0.1 \%$ ). A possible explanation for this discrepancy could be that the pre-hospital time is too short for the occurrence of the described adverse effects. Another explanation could be that pre-hospital data were incompletely documented, which was frequently observed in our study. The chosen design (retrospective EPR study) may also underestimate the true rate of adverse effects [34]. Recently, there is a lot of debate and research ongoing in the call for alternative spinal motion restriction. There are many side effects known of spinal immobilisation, and researchers recently came up with evidence that cervical spine movement was up to four times as high during extrication by EMS as by controlled self-extrication [35]. To reduce the adverse effects caused by spinal immobilisation, the National Association of EMS physicians (NAEMSP) and the American College of Surgeons Committee on Trauma (ACS-COT) have published a position paper in 2013. In this paper they describe that the utilization of backboards for spinal immobilization during transport should be judicious and not be used at all times. A professional consideration should take into account the benefits as well as the risks [36]. 
Table 7 Distracting injuries according to the PHTLS guidelines, fifth edition, 2003

Long bone fractures

Visceral injury requiring surgical consultation

Large laceration

Degloving or crush injury

Large burns

Any other injury producing acute functional impairment

Finally, we found a high number of patients with symptoms (determined by EMS staff) of SCI in our study compared with a large European cohort study (5.7 versus $1.8 \%$, respectively) [37]. Nevertheless, it is difficult to draw comparisons, for while EMS staff can detect symptoms of SCI such as muscle weakness, paralysis or altered sensation, numbness, tingling or loss of sensation in hands, fingers, feet or toes [38], it lacks a validated instrument to uniformly detect symptoms of SCI. The Emergency Department (ED) uses standards of the American Spinal Injury Association (ASIA-ICLOS) to assess damage to the spinal cord of patients. These standards are not applicable in the pre-hospital setting for practical reasons. Furthermore, the EPR of the EMS does not state that documenting symptoms of SCI is mandatory. This means the number of symptoms of SCI in the pre-hospital setting might be overor underestimated in our study. For future research it is necessary to develop a validated and uniform instrument to measure symptoms of SCI in the pre-hospital setting. This enables clinicians to monitor symptoms of SCI over time and to see whether the patient shows signs of deterioration or improvement. This knowledge can contribute to the evaluation of the effectiveness of the current (pre) hospital spinal immobilisation guidelines and improve the quality of care.

This study has some limitations. Data were obtained from only one of the 25 EMS organisations in the Netherlands and may not be representative for spinal immobilisation care in other regions than Gelderland-Zuid. To overcome this limitation, we used a large sample size and we included both rural and urban areas. Despite the regional differences in training and education, all 25 EMS organisations follow a national protocol and their staff is certified by an independent national organisation (Dutch Ambulance Institute).

Another limitation is that we were not able to demonstrate the appropriate use of and adherence to the spinal immobilisation protocol as outlined in Fig. 1 because we did not include patients after blunt trauma who did not receive spinal immobilisation. Furthermore the final outcome of spinal immobilised patients is unknown. Only suspected cases are described and we were not able to compare the patients with suspected spinal column injury with patients in which actual vertebral column/cord injury was diagnosed.

A final limitation concerns the fact that data on a number of variables were incorrectly recorded or missing in the EPR. Adequate documentation by ambulance staff is not only vital for the provision of good care, but also important for trauma research $[33,39,40]$. The lack of adequate EMS documentation has been previously reported [40]. We tried to minimise information bias by analysing the coded data in combination with free text fields. Based on the results of our study, we recommend a revision of the EMS documentation protocol in order to improve the collection of data for research purposes.

\section{Conclusions}

This retrospective observational study described the characteristics and pre-hospital management of patients who received spinal immobilisation by EMS staff. Suspicion of spinal column injuries was documented in $37.2 \%$ of the patients and in $5.7 \%$ of the patients EMS staff reported symptoms of SCI. The combination of a rigid collar, spine board with straps and head blocks (full spinal immobilisation) was used in $84 \%$ of the patients. The remaining $16 \%$ received an alternative or incomplete form of spinal immobilisation. Although one-third of the patients showed signs of head injury and $7 \%$ of these patients had signs of increased ICP, we could not demonstrate the removal of the rigid collar in cases of increased ICP (as advised by PHTLS).

Evident complications of spinal immobilisation were described in less than $2 \%$ of the patients by EMS staff. A reduction in the number of patients who require spinal immobilisation can be achieved by clarifying the term 'distracting injury'. Pain scores were under-recorded by the EMS staff in this cohort.

Finally, attention should be given to improving the process of data registration to make data more reliable. Good documentation is fundamental to scientific research. Therefore, management and staff of the ambulance services should be encouraged to improve their digital records to contribute to future pre-hospital research.

Acknowledgments We would like to thank Theo Liskamp, Wim Heutz, Arjan de Kreek and Peter de Vries for their contributions to this study.

\section{Compliance with ethical standards}

According to the Dutch Central Committee on Research Involving Human Subjects (http://www.ccmo.nl/nl/niet-wmo-onderzoek), retrospective research/patient file research does not fall under the scope of the WMO. Therefore, ethical approval by a healthcare ethics committee was not needed. 
Conflict of interest The authors of this article report no conflicts of interest.

Open Access This article is distributed under the terms of the Creative Commons Attribution 4.0 International License (http://creativecommons.org/licenses/by/4.0/), which permits unrestricted use, distribution, and reproduction in any medium, provided you give appropriate credit to the original author(s) and the source, provide a link to the Creative Commons license, and indicate if changes were made.

\section{References}

1. Toscano J. Prevention of neurological deterioration before admission to a spinal cord injury unit. Paraplegia. 1988;26(3):143-50. doi:10.1038/sc.1988.23.

2. Hauswald M, Ong G, Tandberg D, Omar Z. Out-of-hospital spinal immobilization: its effect on neurologic injury. Acad Emerg Med. 1998;5(3):214-9.

3. Levi AD, Hurlbert RJ, Anderson P, et al. Neurologic deterioration secondary to unrecognized spinal instability following traumaa multicenter study. Spine (Phila Pa 1976). 2006;31(4):451-8. doi:10.1097/01.brs.0000199927.78531.b5.

4. Muckart DJ, Bhagwanjee S, van der Merwe R. Spinal cord injury as a result of endotracheal intubation in patients with undiagnosed cervical spine fractures. Anesthesiology. 1997;87(2):418-20.

5. Ravichandran G, Silver JR. Missed injuries of the spinal cord. Br Med J (Clin Res Ed). 1982;284(6320):953-6.

6. Kraus JF, Franti CE, Riggins RS, Richards D, Borhani NO. Incidence of traumatic spinal cord lesions. J Chronic Dis. 1975;28(9):471-92.

7. Hasler RM, Exadaktylos AK, Bouamra O, Benneker LM, Clancy M, Sieber R, Zimmermann H, Lecky F. Epidemiology and predictors of cervical spine injury in adult major trauma patients: a multicenter cohort study. J Trauma Acute Care Surg (JID 101570622. 0627).

8. Hasler RM, Exadaktylos AK, Bouamra O, Benneker LM, Clancy M, Sieber R, Zimmermann H, Lecky F. Epidemiology and predictors of spinal injury in adult major trauma patients: European cohort study. Eur Spine J Off Publ Eur Spine Soc Eur Spinal Deform Soc Eur Sect Cerv Spine Res Soc (JID 9301980. 2011).

9. American College of Surgeons. Committee on trauma. ATLS: advanced trauma life support for doctors (student course manual), 8th ed. Chicago: American college of surgeons; 2008

10. McSwain NE, Frame S, Salomone JP, editors. PHTLS: Basic and advanced prehospital trauma life support, 5th ed. St. Louis: Mosby, Inc.; 2003.

11. Dutch National protocol ambulance care. http://www.ambulancezorg.nl/nederlands/pagina/2037/lpa-7-2.html. Accessed 2013.

12. Jedlicka DS. A comparison of the effects of two methods of spinal immobilization on respiratory effort in the older adult. Ohio State University. 1997.

13. Totten VY, Sugarman DB. Respiratory effects of spinal immobilization. Prehosp Emerg Care. 1999;3(4):347-52.

14. Hunt K, Hallworth S, Smith M. The effects of rigid collar placement on intracranial and cerebral perfusion pressures. [-6]. 2001 [-0003-2409 (print); -0003-2409 (linking)].

15. Hunt K, Bajekal R, Calder I, Meacher R, Eliahoo J, Acheson JF. Changes in intraocular pressure in anesthetized prone patients. $\mathrm{J}$ Neurosurg Anesthesiol. 2004;16(4):287-90.
16. Mobbs RJ, Stoodley MA, Fuller J. Effect of cervical hard collar on intracranial pressure after head injury. ANZ J Surg. 2002;72(6):389-91.

17. Stelfox H, Velmahos G, Gettings E, Bigatello L, Schmidt U. Computed tomography for early and safe disconnection of cervical spine immobilization in obtunded multiply injured patients. J. Trauma [-3]. 2007;63:630-36.

18. Kwan I, Bunn F. Effects of prehospital spinal immobilization: a systematic review of randomized trials on healthy subjects. Prehosp Disaster Med. 2005;20(1):47-53.

19. Kwan I, Bunn F, Roberts I. Spinal immobilisation for trauma patients. Cochrane Database Syst Rev. 2001;2(2):CD002803. doi:10.1002/14651858.CD002803.

20. Slagel SA, Skiendzielewski JJ, McMurry FG. Osteomyelitis of the cervical spine: Reversible quadraplegia resulting from philadelphia collar placement. Ann Emerg Med. (JID 8002646. 0920).

21. Ben-Galim P, Dreiangel N, Mattox KL, Reitman CA, Kalantar SB, Hipp JA. Extrication collars can result in abnormal separation between vertebrae in the presence of a dissociative injury. J Trauma (JID 0376373. 0922).

22. Papadopoulos MC, Chakraborty A, Waldron G, Bell BA. Lesson of the week: exacerbating cervical spine injury by applying a hard collar. BMJ Clin Res Ed; 1999 (JID 8900488. 0920(0959-535).

23. Barkana YF, Stein MF, Scope AF, et al. Prehospital stabilization of the cervical spine for penetrating injuries of the neck-is it necessary? Injury (JID 0226040. 0720).

24. Abram S, Bulstrode C. Routine spinal immobilization in trauma patients: What are the advantages and disadvantages? [-4]. 2010 [-1479-666X (print); -1479-666X (linking)].

25. Bijur PE, Latimer CT, Gallagher EJ. Validation of a verbally administered numerical rating scale of acute pain for use in the emergency department. Acad Emerg Med Off J Soc Acad Emerg Med (JID 9418450. 0911).

26. Bauer D, Kowalski R. Effect of spinal immobilization devices on pulmonary function in the healthy, nonsmoking man. Ann Emerg Med. 1988;17(9):915-8.

27. Hsieh H, Shannon S. Three approaches to qualitative content analysis. Qual Health Res. 2005;15(9):1277-88.

28. Heffernan DS, Schermer CR, Lu SW. What defines a distracting injury in cervical spine assessment? J Trauma. 2005;59(6):1396-9.

29. Domeier R, Frederiksen S, Welch K. Prospective performance assessment of an out-of-hospital protocol for selective spine immobilization using clinical spine clearance criteria. Ann Emerg Med. 2005;46(2):123-31.

30. Konstantinidis A, Plurad D, Barmparas G, et al. The presence of nonthoracic distracting injuries does not affect the initial clinical examination of the cervical spine in evaluable blunt trauma patients: a prospective observational study. J Trauma. 2011;71(3):528-32.

31. Dahlquist RT, Fischer PE, Desai H, et al. Femur fractures should not be considered distracting injuries for cervical spine assessment. Am J Emerg Med. 2015;33(12):1750-4.

32. Cason B, Rostas J, Simmons J, Frotan MA, Brevard SB, Gonzalez RP. Thoracolumbar spine clearance: clinical examination for patients with distracting injuries. J Trauma Acute Care Surg. 2016;80(1):125-30.

33. Harkins S. Documentation: why is it so important? Emerg Med Serv. 2002;31(10):89-90, 93-4.

34. Zegers M, de Bruijne MC, Wagner C, et al. Design of a retrospective patient record study on the occurrence of adverse events among patients in dutch hospitals. BMC Health Serv Res. 2007;7(1):1. 
35. Dixon M, O'Halloran J, Cummins NM. Biomechanical analysis of spinal immobilisation during prehospital extrication: a proof of concept study. Emerg Med J. 2014;31(9):745-9.

36. White CC, 4th, Domeier RM, Millin MG. Standards and Clinical Practice Committee, National Association of EMS Physicians. EMS spinal precautions and the use of the long backboard-resource document to the position statement of the national association of EMS physicians and the American College of Surgeons committee on trauma. Prehosp Emerg Care. 2014;18(2):306-14.

37. Hasler RM, Exadaktylos AK, Bouamra O, Benneker LM, et al. Epidemiology and predictors of spinal injury in adult major trauma patients: European cohort study. Eur Spine J Off Publ
Eur Spine Soc Eur Spinal Deform Soc Eur Sect Cerv Spine Res Soc. 2011 (JID 9301980).

38. Bernhard M, Gries A, Kremer P, Böttiger BW. Spinal cord injury (SCI)—prehospital management. review. Resuscitation. 2005.

39. Laudermilch DJ, Schiff MA, Nathens AB, Rosengart MR. Lack of emergency medical services documentation is associated with poor patient outcomes: a validation of audit filters for prehospital trauma care. J Am Coll Surg. 2010;210(2):220-7.

40. Staff T, Sovik S. A retrospective quality assessment of pre-hospital emergency medical documentation in motor vehicle accidents in south-eastern Norway. Scand J Trauma Resusc Emerg Med. 2011;19:20-7241-19-20. 